\title{
Extended Spectrum $\beta$-Lactamases among Gram-negative bacteria from an Egyptian pediatric hospital: a two-year experience
}

\author{
Maysaa El Sayed Zaki \\ Clinical Pathology, Mansoura University, Egypt
}

\begin{abstract}
Background Knowledge of the anti-microbial susceptibility pattern of common pathogens in a given area helps to inform the choice of empirical antibiotic therapy.

The aim of this study was to determine the existence and to describe the characteristics of extended spectrum $\beta$-lactamase (ESBLs) in a pediatric hospital in Mansoura University, Egypt, to aid in the choice of empirical antibiotic therapy.

Method: Between January 2005 and December 2006, blood samples were collected from children suspected to have nosocomial infections in a pediatric hospital in Mansoura. The gram negative isolates were identified, tested for antimicrobial susceptibility and analyzed for presence of ESBL.

Results: A total of 1,600 children with suspected septicemia were studied. Gram negative septicemia was identified in 816 cases (45\%). The commonest isolated gram negative bacilli was Klebsiella species (38.2\%) followed by Enterobacter species (32.4), Serratia species (16.2\%) and Burkholderia cepacia (10.3\%). The highest susceptibility was for imipenem (74.3\%) followed by gentamicin $(70.8 \%)$, cefoperazone $(64.5 \%)$ and cefotaxime $(63.2 \%)$. The highest resistant rate was for cefazolin and ampicillin/sulbactam (75.5\% for each), followed by cefuroxime $70.3 \%$ and ceftriaxone (63.5\%). The ESBL was found in $44.5 \%$ for cefotaxime and $50 \%$ for ceftazidime by double discs method.

Conclusion: This study highlights the emergence of antibiotic resistant gram negative bacilli in a pediatric hospital with special emphasis on extended $\beta$-lactamase resistant strains. Our results show that the most appropriate antibiotics to be used for empirical therapy are amikacin and gentamicin.
\end{abstract}

Key Words: Gram negative sepsis, beta-lactamase resistant strains phenotype study.

J Infect Developing Countries 2007; 1(3):269-274.

Received 28 July 2007 - Accepted 25 September 2007.

Copyright @ 2007 Zaki. This is an open access article distributed under the Creative Commons Attribution License, which permits unrestricted use, distribution, and reproduction in any medium, provided the original work is properly cited.

\section{Introduction}

Sepsis, a common cause of pediatric morbidity and mortality, is a systemic inflammatory response (SIR) to infection. Children with septicemia present with fever, difficult breathing, tachycardia, malaise, inability to feed or lethargy, but those with asymptomatic bacteraemia tend to show no obvious signs of illness [1]. Deaths from pediatric septicemia are likely to be higher in low-income settings.

In a hospital setting, the proportion of septic infections due to gram-negative bacteria (GNB) varies between $30 \%$ and $80 \%$ whereas infections due to gram-positive bacteria vary between $6 \%$ and $24 \%$ [2].

Prompt diagnosis and effective treatment is necessary to prevent death and complications from septicaemia. The results of bacteriological cultures and antibiotic susceptibility tests take about a week, necessitating initial empirical treatment of suspected septicaemia. Knowledge of epidemiological and anti-microbial susceptibility patterns of common pathogens in a given area helps to inform the choice of antibiotics $[3,4]$.

Extended spectrum $\beta$-lactamase (ESBL) enzymes have been reported in a number of species in gram-negative bacteria. The ESBL enzymes are usually plasmid mediated and are capable of hydrolyzing and inactivating a wide variety of $\beta$-lactams, including third-generation cephalosporins, penicillin and aztreonam, but are susceptible to $\beta$-lactamase inhibitors such as clavulanate, sulbactam and tazobactam $[5,6]$. However, not all ESBL producers are inhibited by those inhibitors, especially for those ESBL belonging to groups other than SHV or TEM betalactamases. Many ESBL producers also carry other genes that confer resistance to other antimicrobial agents such as aminoglycosides and fluoroquinolones $[7,8]$. 
Extensive use of broad spectrum antibiotics, prolonged hospitalization, indwelling devices and severe underlying diseases have been reported as factors that have led to the spread of ESBLs and difficulties in managing severe infections in many parts of the world $[9,10]$. Treatment of ESBLproducing bacterial infections can place an added constraint on already overburdened health systems in developing countries. ESBL producing organisms are reported to account for a significant proportion of intensive care infections [11], and problems related to ESBLs have led to limited as well as expensive treatment options, and have impacted negatively on clinical outcomes [12]. Nosocomial infections due to ESBL producing organisms have been known to cause high mortality [13].

The present study was conducted to highlight the bacteriological profile of gram negative sepsis and the pattern of antibiotics susceptibility tests in a pediatric hospital in Mansoura, Egypt, over a two-year period.

\section{Materials and Methods}

The study included all consecutive blood cultures in children aged 0 to 15 years admitted to the Mansoura University Pediatric Hospital from January 2005 to December 2006. A total of 1600 patients were screened. The indication for blood cultures were clinical features judged by the attending clinician to be indicative of sepsis, especially fever without localized lesion.

\section{Bacteriological culture}

Blood culture: Specimens for microbiologic investigations were divided equally and inoculated into aerobic bottles (BACTEC PEDS plus/F) and anaerobic bottles (BACTEC Plus/F). BACTEC 9050 (Becton Dickinson) blood culture system was used for aerobic and anaerobic culture. The flagged positive bottles were selected for Gram staining of an aspirate of the broth. Gram negative bacilli isolated from each patient were chosen for further studies. Subcultures were performed on fresh blood agar and incubated aerobically at $37^{\circ}$ C. Identification of bacterial isolates was performed by Microscan WalkAway system. The bottles were flagged negative after 5 days and sub-cultured under the same conditions as the positive bottles before being discarded as negative [14].
Identification by MicroScan WalkAway®-96 System (Dade® Behring, Inc., West Sacramento, CA) using routine testing panels

We used the Microscan identification system for identification of recovered microorganisms. Modified conventional and chromogenic tests were used for the identification of fermentative and nonfermentative gram-negative bacilli. Identification was based on detection of $\mathrm{pH}$ changes, substrate utilization and growth in the presence of antimicrobial agents after 16 to 42 hours of incubation at about $35^{\circ} \mathrm{C}$.

\section{Susceptibility testing}

Microscan System: Antibiotic susceptibilities were determined with commercial dehydrated panels provided by Dade Behring MicroScan (Sacramento, Calif.) according to the manufacturer's recommendations, and interpreted according to NCCLS criteria [15]. The panels were selected on the basis of the concentrations and types of $\beta$-lactam drugs in the panel from among a number of panels available to the routine clinical laboratory [16,17]. The antibiotics aztreonam, cefazolin, cefoperazone, cefotaxime, ceftazidime, ceftriaxone, and cefuroxime were selected as they are potentially useful for the detection and differentiation of the $\beta$-lactamases present in GNB strains.

Double discs for determining the Extendedspectrum B-lactamases (ESBL): A double disc confirmatory test was performed using cefotaxime, cefotaxime-clavulanate, ceftazidime and ceftazidime-clavulanate antimicrobial discs and the results were read as recommended by the NCCLS [18].

\section{Results}

A total of 1,600 children with suspected septicemia were studied. Gram negative septicemia was identified in 816 cases (45\%). They were 500 males (61.3\%) and 316 females (38.8\%). The majority of cases with sepsis were in the intensive care unit (ICU) $(25 \%)$ and neonatal ward $(20 \%)$, (Figure 1). The main underlying diseases were bronchopneumonia (31.7\%), congenital heart diseases (10\%), independent diabetes mellitus (8.3\%), preterm birth (8.3\%), acute leukemia and lymphoma (6.7\%), and surgical operations $(6.7 \%)$. The majority of cases were in age groups $<1$ month (less than 1 month); 
1-12months and >1year-5years, which accounted for $33.3 \%, 25 \%$ and $21.7 \%$ respectively (Figure 2).
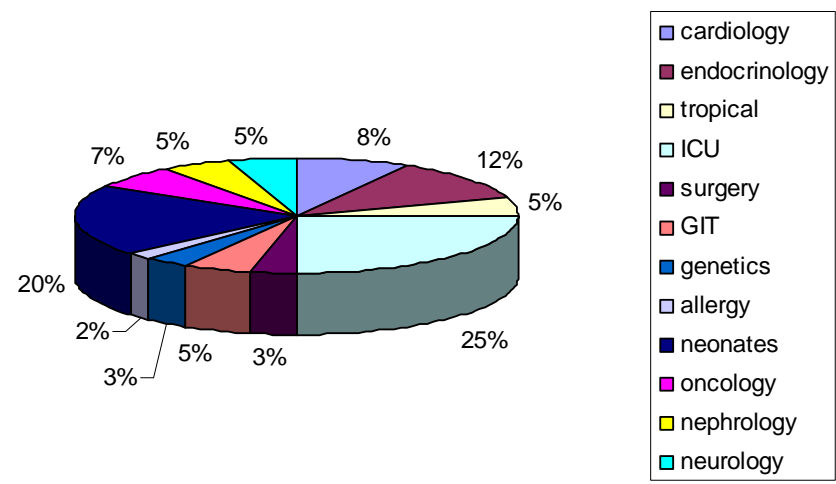

Figure 1. Ward Distribution of isolated GNB.

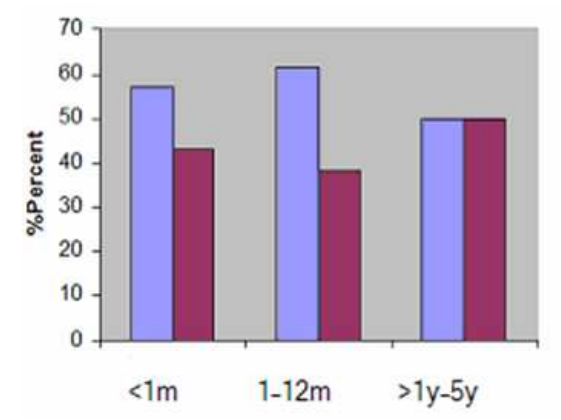

Figure 2. Age distribution of patients.

(m, month; $y$, year)

\section{Bacteriological Profile of GNB Isolates}

The commonest isolated gram negative bacilli were Klebsiella species (38.3\%), Enterobacter spp. (32.4), Serratia spp. (16.2\%) and Burkholderia cepacia (10.3\%) (Table 1).

Table 1. Isolated Bacterial Species.

\begin{tabular}{lcc}
\hline Organism & $\begin{array}{c}\text { No. of } \\
\text { isolates }\end{array}$ & $\%$ \\
\hline Kelbsiella species & 312 & 38.3 \\
K.pneumoniae & 200 & 24.5 \\
K.oxytoca & 112 & 13.8 \\
Enterobacter spp & 264 & 32.4 \\
E.aerogenes & 164 & 20.1 \\
E.clocae & 100 & 12.3 \\
Serratia spp & 132 & 16.2 \\
S.marcescens & 80 & 9.8 \\
S.rubidea & 52 & 6.4 \\
Burkholderia cepacia & 84 & 10.3 \\
Shigella spp & 12 & 1.5 \\
E.coli & 12 & 1.5 \\
\hline Total & 816 & 100 \\
\hline
\end{tabular}

The highest susceptibility for the isolated strains was for imipenem $(74.3 \%)$ followed by gentamicin $(70.8 \%)$, cefoperazone $(64.5 \%)$ and cefotaxime $(63.2 \%)$. The highest rate of resistant strains was for cefazolin and ampicillin/sulbactam (75.5\% for each), followed by cefuroxime $70.3 \%$ and ceftriaxone (63.5\%) (Table 2).

Table 2. Gram negative bacilli susceptibility pattern.

\begin{tabular}{lcc} 
& \multicolumn{2}{c}{$\begin{array}{c}\text { Susceptible } \\
\text { Strains }\end{array}$} \\
\cline { 2 - 3 } Antibiotics & $\mathbf{N}$ & $\%$ \\
\hline Aztreonam & 322 & 39.5 \\
Cefazolin & 200 & 24.5 \\
Cefoperazone & 526 & 64.5 \\
Cefotaxime & 516 & 63.2 \\
Ceftazidime & 316 & 38.7 \\
Ceftriaxone & 298 & 36.5 \\
Cefuroxime & 242 & 29.7 \\
Ampicillin/sulbactam & 200 & 24.5 \\
Amikacin & 410 & 50.2 \\
Gentamicin & 578 & 70.8 \\
Imipenem & 606 & 74.3 \\
\hline
\end{tabular}

As shown in Table 3, out of the 312 Klebsiella species isolated, $23.1 \%$ were resistant to imipenem; the highest resistance was to ampicillin/sulbactam (69.9\%) and cefazolin (68.9\%).

Of the 264 Enterobacter spp. the highest susceptibility was for gentamicin and imipenem $(68.2 \%)$, while this species was associated with high resistance to the three classes of cephalosporins (76.9\%-86\%).

Of the 132 Serratia species, high susceptibility was to gentamicin and imipenem (81.8\%) and cefotaxime $72.7 \%$. In contrast, $72.7 \%$ of these isolates were resistant to cefuroxime. Among 84 Burkholderia cepacia isolates studied, $68.2 \%$ were susceptible to gentamicin and imipenem but showed high $(76.9 \%)$ resistance to ceftriaxone. ESBLs were detected in $44.5 \%$ of all GNB for cefotaxime and $50 \%$ for ceftazidime by double discs method (data not shown). 


\section{Discussion}

We observed high resistance rates among the commonly isolated GNB to the beta-lactam antibiotics. Klebsiella and Enterobacter spp. were highly resistant to the third generation cephalosporins which are commonly used to treat nosocomial infections and are frequent first-line drugs for patients admitted to the hospital. In fact certain centers use ceftriaxone and ceftazidime as empirical therapy for patients with febrile neutropenia $[19,20]$. Burkholderia spp. appears as important as GNB isolated from our patients as they also had similar patterns of resistance to beta-lactam antibiotics.

Table 3. Antibiotic susceptibility of isolated species.

\begin{tabular}{|c|c|c|c|c|}
\hline & \multicolumn{4}{|c|}{ Susceptibility Pattern } \\
\hline & $\begin{array}{l}\frac{0}{0} \\
\frac{0}{2} \\
\frac{0}{20} \\
\frac{0}{d} \\
\frac{1}{2}\end{array}$ & 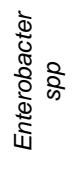 & $\begin{array}{l}\frac{0}{2} \\
\frac{0}{2} \\
\stackrel{0}{\pi} \\
\frac{\pi}{2} \\
\infty \\
\infty\end{array}$ & $\begin{array}{l}\frac{\pi}{0} \\
\frac{0}{0} \\
\frac{5}{2} \\
\frac{5}{3} \\
0\end{array}$ \\
\hline \multirow[t]{2}{*}{$\mathbf{N}$} & 312 & 264 & 132 & 84 \\
\hline & $\mathrm{S} \%$ & $\mathrm{~S} \%$ & $\mathrm{~S} \%$ & $\mathrm{~S} \%$ \\
\hline Aztreonam & 43.3 & 20.1 & 37.1 & 62.9 \\
\hline Cefazolin & 31.1 & 14.0 & 50.0 & 50.0 \\
\hline Cefoperazone & 35.3 & 23.1 & 46.2 & 53.8 \\
\hline Cefotaxime & 43.3 & 23.1 & 72.7 & 27.3 \\
\hline Ceftazidime & 35.3 & 23.1 & 46.2 & 46.2 \\
\hline Ceftriaxone & 42.0 & 23.1 & 55.3 & 23.1 \\
\hline Cefuroxime & 42.0 & 18.2 & 27.3 & 72.7 \\
\hline Ampicillin/sulbactam & 30.1 & 9.8 & 40.2 & 40.2 \\
\hline Amikacin & 50.0 & 20.0 & 40.0 & 40.0 \\
\hline Gentamicin & 69.9 & 68.2 & 81.8 & 68.2 \\
\hline Imipenem & 76.9 & 68.2 & 81.8 & 68.2 \\
\hline
\end{tabular}

ESBL production appears to be a major mechanism of ceftazidime resistance in strains of GNB as $44.6 \%$ were found to be ESBL producers by double-disc screening test. Klebsiella spp. Is a well-known producer and has been previously known to cause outbreaks in ICUs [21] in other countries.

The results of this study indicate that broth micro dilution panels currently available to the clinical laboratory can provide a vehicle for the detection of $\beta$-lactamases capable of producing hidden resistance to expanded-spectrum cephalosporins and aztreonam in isolates of $E$. coli, K. pneumoniae, and K. oxytoca. Similar conclusions were provided by Moland et al. [22] and Wiegand et al. [23].

Based on data from this study, some guidelines regarding therapeutic choices have emerged. There is a clear indication that GNB isolated from the pediatric hospital are less susceptible to the third generation cephalosporins. Empirical therapy of a patient known to be infected with GNB in our hospital in whom sensitivity information is pending or in whom clinical parameters suggest failure while receiving third generation cephalosporins, may dictate a change of treatment to imipenem or gentamicin or amikacin. The high sensitivity of GNB to imipenem indicates the absence of selective pressure since the drug is rarely prescribed. It is also of note that despite heavy usage, amikacin has retained good susceptibility rates due to its reduced use as empirical therapy and has not exhibited crossresistance with ceftazidime. Thus the role of amikacin in combination therapy for the patients admitted to the pediatric hospital appears to be justified.

Earlier reports have suggested the relation between bacterial resistance with widespread antibiotic use $[24,25,26]$. The most common sites in our study acquiring GNB sepsis were the ICU followed by neonatal units. The highest rate of resistance is often seen in areas with the highest rates of antibiotic prescriptions $[27,28]$. Strategies to control increasing antimicrobial resistance rates include limiting the use of antibiotics as reported by Meyer et al. [29] where restriction of ceftazidime use successfully overcame an outbreak of resistant Klebsiella pneumoniae. Restriction and rotation of broad spectrum antibiotics were also found to be associated with a reduction of ventilator associated pneumonia [13], another major problem in ICUs. Although it may appear theoretically simple to address the problem of increasing resistance by withholding or restricting antibiotic use, this approach is probably not 
practical when dealing with patients where initial empirical broad spectrum therapy is clearly required while awaiting culture results.

Studies have shown that inadequate antibiotic therapy or a delay in initiating appropriate therapy is associated with increased risk of mortality in patients admitted to the ICU [30,31]. Namias et al. [16] reported on the practice of using an imipenem-gentamicin combination for empirical treatment of sepsis in his surgical ICU followed by de-escalation to a more narrow-spectrum antibiotic when culture results were available, usually after 48 to 72 hours. The role of strict infection control practices can not be over-emphasized, especially when cross-contamination is a major mode of acquisition of multi-resistant bacteria [32]. Additional steps to address the growing threat of antibiotic resistant GNB include monitoring resistance trends in major pathogens on a continuous basis and modification of institutional antibiotic formularies as required. This study highlights the emergence of antibiotic resistant GNB in a pediatric hospital in Egypt with special emphasis on extended beta-lactamase resistant strains. It seems that the most appropriate antibiotics to be used for empirical therapy are amikacin and gentamicin.

\section{References}

1. Rackow EC (1986) Clinical definition of sepsis and septic shock. In: Sibbald WJ, Sprung CL (eds) New horizons: perspectives of sepsis and septic shock. Society of critical care medicine, Fullerton, PP 1-9.

2. Glauser MP, Zanetti G, Baumgartner JD, Cohen J (1991) Septic shock: pathogenesis. Lancet 338: 732-736.

3. Hague RA, Eastman EJ, Lee RE, Cant AJ (1993) Resolution of hepatic abscess after interferon gamma in chronic granulomatous disease. Arch Dis child 69:443-5.

4. Weber MW, Carlin JB, Gatchalian S, Lehmann D, Muhe L, Mulholland EK, WHO Young Infants Study Group (2003) Predictors of neonatal sepsis in developing countries. Pediatr Infect Dis 22:711-7.

5. Sturenburg E, Mack D (2003) Extended-spectrum betalactamases: implications for the clinical microbiology laboratory, therapy, and infection control. J Infect 47:273295.

6. Gniadkowski M (2001) Evolution and epidemiology of extended-spectrum beta-lactamases (ESBLs) and ESBLproducing microorganisms. Clin Microbiol Infect 7:597608.

7. Spanu T, Luzzaro F, Perilli M, Amicosante G, Toniolo A, Fadda G(2002) Occurrence of extended-spectrum betalactamases in members of the family Enterobacteriaceae in Italy: implications for resistance to beta-lactams and other antimicrobial drugs. Antimicrob Agents Chemother 46:196-202.

8. Sekowska A, Janicka G, Klyszejko C, Wojda M, Wroblewski M, Szymankiewicz M (2002) Resistance of Klebsiella pneumoniae strains producing and not producing ESBL (extended-spectrum beta-lactamase) type enzymes to selected non-beta-lactam antibiotics. Med Sci Monit 8:BR100-4.

9. Nathisuwan S, Burgess DS, Lewis JS (2001) Extendedspectrum beta-lactamases: epidemiology, detection and treatment. Pharmacotherapy 21:920-928.

10. Lin MF, Huang ML, Lai SH (2003) Risk factors in the acquisition of extended-spectrum beta-lactamase Klebsiella pneumoniae: a case-control study in a district teaching hospital in Taiwan. J Hosp Infect 53:39-45.

11. Paterson DL, Ko WC, Von Gottberg A, Mohapatra S, Casellas JM, Goossens H, Mulazimoglu L, Trenholme G, Klugman KP, Bonomo RA, Rice LB, Wagener MM, McCormack JG, Yu VL (2004) International prospective study of Klebsiella pneumoniae bacteremia: implications of extended-spectrum beta-lactamase production in nosocomial Infections. Ann Intern Med 140:26-32.

12. Menashe G BA, Yagupsky P, Peled N, Gilad J, Fraser D, Riesenberg K, Schlaeffer F (2001) Clinical significance and impact on mortality of extended-spectrum beta lactamase-producing Enterobacteriaceae isolates in nosocomial bacteremia. Scand J Infect Dis 33:188-193.

13. Ho PL, Chan WM, Tsang KW, Wong SS, Young K (2002) Bacteremia caused by Escherichia coli producing extended-spectrum beta-lactamase: a case-control study of risk factors and outcomes. Scand J Infect Dis 34:567573.

14. Connie RM, Manuselis G (2000) Bacteremia. Textbook of Diagnostic. Microbiology. 2:997-1008.

15. National Committee for Clinical Laboratory Standards (1997) Methods for dilution antimicrobial susceptibility tests for bacteria that grow aerobically. Approved standard M7-A4. Wayne, Pa: National Committee for Clinical Laboratory Standards.

16. National Committee for Clinical Laboratory Standards (1997) Performance standards for antimicrobial disk susceptibility tests. Approved standard M2-A6. Wayne, Pa: National Committee for Clinical Laboratory Standards.

17. Weill FX, Demartin M, Tande D, Espie E, Rakotoarivony I, Grimont PA (2004) SHV-12-like extended-spectrumbeta-lactamase-producing strains of Salmonella enterica serotypes Babelsberg and Enteritidis isolated in France among infants adopted from Mali. J Clin Microbiol 42:2432-2437.

18. National Committee for Clinical Laboratory Standards (2000) Performance Standards for Antimicrobial Disc Susceptibility- Seventh Edition: Approved Standard M2A7. NCCLS, Wayne, PA.

19. Pitout JDD, Sanders CC, Sanders WE (1997) Antimicrobial resistance with focus on B-lactam resistance in Gram negative bacilli. American $\mathrm{J}$ of Med 103, 51-9.

20. Ariffin $\mathrm{H}$ et al. (2001) Single daily ceftriaxone plus amikacin vs. thrice daily ceftazidime plus amikacin as empirical therapy for children with febrile neutropenia. $J$ child health $37,38-43$. 
21. Parasakthi $\mathrm{N}$ et al. (2000) Epidemiology and molecular characterization of nosocomially transmitted multi-drug resistant Klebsiella pneumoniae. Int J Infect Dis 4:123-4.

22. Moland E S, Christine C. Sanders C C, and Thomson K S (1998) Can Results Obtained with Commercially Available MicroScan Microdilution Panels Serve as an Indicator of $\beta$-Lactamase Production among Escherichia coli and Klebsiella Isolates with Hidden Resistance to Expanded Spectrum Cephalosporins and Aztreonam? J Clin Microbiol 36: 2575-2579.

23. Wiegand I, Geiss H K, Mack D, Stürenburg E, and Seifert H (2007) Detection of Extended-Spectrum BetaLactamases among Enterobacteriaceae using Semiautomated Microbiology Systems and Manual Detection Procedures J Clin Microbiol 1988-06.

24. Shales DM et al. (1997) Guidelines for the prevention of antimicrobial resistance in hospitals: Joint statement by society for health care epidemiology of America and infectious society of America. Infect Control Hosp Epidemiol 18:275-291.

25. McGown JE, Gerding D (2000) Does antibiotic restriction prevent resistance? New Horiz 4:370-76.

26. Rahal JJ, Urban C, Segal-Maurer S (2002) Nosocomial antibiotic resistance in multiple gram negative species: experience at one hospital with squeezing the resistance balloon at multiple sites. Clin Infect Dis, 34:499-503.

27. Meyer K S, Urban C, Eagan JA, Berger BJ, Rahal JJ (1993) Nosocomial out breaks of Klebsiella infection resistant to the third generation cephalosporins. Ann Intern Med 119:353-58.
28. Grason D et al. (2000) Rotation and restricted use of antibiotics in a medical intensive care unit. Am J Respir Crit Care Med 162:837-43.

29. Luna CM, Vujarich $P$, Niederman MS et al. (1997) Impact of broncho alveolar lavage data on the therapy and outcome of ventilator associated pneumonia. Chest 111:676-85.

30. Alvarez-Lerma F (1996) Modification of empiric antibiotic treatment in patients with pneumonia acquired in the intensive care unit. Intensive Care Med 22:387-94.

31. Namias $\mathrm{N}$ et al. (1998) Empiric therapy of sepsis in the surgical intensive care unit with broad spectrum antibiotics for 72 hours does not lead to the emergence of resistant bacteria. J Trauma Infect Crit Care 45:887-91.

32. Harris A, Torres-Viera C, Venkataraman L, DeGirolami $P$, Samore M, Carmeli Y (1999) Epidemiology and clinical outcomes of patients with multi-resistant Pseudomonas aeruginosa. Clin Infect Dis 28:1128-33.

Corresponding Author: Maysaa El Sayed Zaki, EgyptMansoura- El Gomhoria street - Mansoura UniversityClinical Pathology Department - Dr. Maysaa El Sayed Zaki, Telephone: 00-20-50-225-88-77, e-mail: may_s65@hotmail.com

Conflict of interest: The authors declare that they have no conflict of interests. 